Methods Data were collected from June 2009 to February 2010, from all elementary and junior high schools located in a northern subprefecture of Ibaraki, $100 \mathrm{~km}$ north of Tokyo (approximate population of 280000$)$. The information included school name, school address, the enrolment number by school, dates of absentees from influenza-like illness (type-A), date of class closure and date of school closure. Impacts of school closure was analysed considering timing of closure, enrolment number, geographical location.

Result All 68 schools responded for study. 8576 out of 23880 (143280 person-months) enrolled school children were infected. We classified these schools into two groups according to date of school closure. The difference of incidence between two groups was estimated with the RR with 95\% CI. The incidence rates in the early and late school closure groups were 71.6 and 64.9 (1/1000 personmonths), respectively. RR was 1.10 (95\% CI 1.05 to 1.15$)$, which was significant.

Conclusion The result would show that the early school closure may have impact to prevent infection number of $\mathrm{A} / \mathrm{H} 1 \mathrm{~N} 1$ influenza.

\section{P1-364 IS SOCIAL CAPITAL IN THE WORKPLACE ASSOCIATED WITH WORK-RELATED INJURY AND DISABILITY? A SYSTEMATIC REVIEW OF THE EPIDEMIOLOGIC LITERATURE}

doi:10.1136/jech.2011.142976f.56

\begin{abstract}
$1,2 \mathrm{~V}$ Kristman, ${ }^{2,3} \mathrm{~A}$ Vafaei. ${ }^{*}$ Dalla Lana School of Public Health, University of Toronto, Toronto, Ontario, Canada; ${ }^{2}$ Centre of Research Expertise in Improved Disability Outcomes, University Health Network, Toronto, Ontario, Canada; ${ }^{3}$ Department of Community Health \& Epidemiology, Queen's University, Kingston, Ontario, Canada
\end{abstract}

Introduction Social capital is defined as resources embedded in social relationships. Social capital in the workplace may include social support, interpersonal trust, respect, and reciprocity; and may occur at both the worker and workplace level. The objective of this study was to determine if social capital in the workplace is associated with work-related injury or disability.

Methods A systematic review of the epidemiologic literature was conducted. Studies were identified from 1990 to 2008 relevant to social capital in the workplace and work-related injury or disability. Identified studies were critically appraised for methodological quality by two qualified independent reviewers. Findings represent a best evidence synthesis of the literature.

Results Sixty-six studies were scientifically reviewed. Forty-two were excluded due to poor methodological quality. The remaining 24 consisted of 14 studies examining the association between social capital in the workplace and work-related injury, eight focused on disability, and two studied both injury and disability. Only two studies included workplace-level social capital, the remaining focused on individual-level worker social capital. Limitations of the literature include unclear social capital, injury and disability definitions, limited study populations, and weak study designs.

Conclusions Limitations preclude stating consistent conclusions. The evidence suggests an association between individual worker social capital and work disability. Group-level social capital may be important in the development of work-related injury and disability. We summarised the literature, highlighted its strengths and weaknesses, and provided suggestions for future work.

\section{P1-365 ASSOCIATION OF DISTANCES TO URBAN GREEN SPACES AND OPEN SPORT SPACES WITH PHYSICAL ACTIVITY IN TEENAGERS OF PORTO, PORTUGAL}

doi:10.1136/jech.2011.142976f.57

\footnotetext{
${ }^{1,2} \mathrm{~A}$ Magalhães, ${ }^{1,2} \mathrm{E}$ Ramos, ${ }^{*}{ }^{1,3} \mathrm{~F}$ Pina. ${ }^{1}$ Department of Hygiene and Epidemiology, Porto Medical School, Porto, Portugal; ' Institute of Public Health, Porto University,
}

Porto, Portugal; ${ }^{3}$ Institute of Biomedical Engineering INEB, Porto University, Porto, Portugal

Introduction It is expected that the proximity to urban green spaces (UGS) and open sport spaces (OSS) can increase physical activity (PA), but their potential is not fully understood.

Objective To examine the association between distances from adolescent's residences to UGS and OSS with the intensity of PA and sports activity.

Methods We evaluated 1489 (51.4\% girls) 17-year-old adolescents living in Porto, Portugal (EPITeen cohort). Residences were georeferenced. Buffers of $250 \mathrm{~m}$ and $500 \mathrm{~m}$ around each space were created and distances to residences were classified in $\leq 250 \mathrm{~m}$ (class 1 ), $>250 \mathrm{~m}$ and $\leq 500 \mathrm{~m}$ (class2) and $>500 \mathrm{~m}$ (class3). Association between distances to spaces and Intensity of PA (adjusted to BMI, and parents' education) and with sports activity (adjusted to parents' education and obesity) were measured using OR and 95\% CI using logistic regression analysis.

Results Considering class1 as reference, in girls the association between distance to UGS and Intensity of PA was $0.99(0.70 ; 1.42)$ for class 2 and $0.81(0.54 ; 1.24)$ for class 3 . Among boys, those results were $0.85(0.58 ; 1.24)$ and $0.69(0.46 ; 1.03)$, respectively. Regarding the association between distance to OSS and intensity of PA, in girls, was $0.75(0.50 ; 1.13)$ for class 2 and $1.14(0.76 ; 1.71)$, for class 3 Among boys, those results were $1.30(0.84 ; 2.01)$ and 1.38 (0.90;2.11), respectively. Similar results were found between distances to UGS and OSS and sports activity.

Conclusion Adolescents closer to UGS and those most far from OSS were more physically active, although in general no statistically significant association was reached.

\section{P1-366 A PROSPECTIVE MIXED METHODS COHORT STUDY TO ASSESS ETHNIC INEQUITIES IN PATIENT SAFETY IN DUTCH HOSPITAL CARE}

doi:10.1136/jech.2011.142976f.58

${ }^{1,2} \mathrm{~F}$ van Rosse, ${ }^{*} \mathrm{M}$ de Bruijne, ${ }^{1} \mathrm{~K}$ Stronks, ${ }^{2} \mathrm{C}$ Wagner, ${ }^{1} \mathrm{M}$ L Essink-Bot. ${ }^{1}$ Academic Medical Center, Amsterdam, The Netherlands, ${ }^{2}$ VU medical center, Amsterdam, The Netherlands

Introduction USA studies showed an increased risk of patient safety incidents in hospital care among ethnic minorities, but in Europe ethnic disparities in patient safety have never been analysed. The present study aims to 1 . Assess the risk of patient safety events in patients of non-western ethnic origin in comparison to Dutch patients 2 . Analyse the determinants of the risk of adverse events in patients of non-western origin, and 3. Explore causal mechanisms in patient-provider interaction.

Methods Prospective mixed methods cohort study in four hospitals. In total, 2000 patients (1000 Dutch, 1000 of any non-Western ethnic origin, age range:45-75 years) will be included. Data on explanatory variables (eg, Dutch language proficiency, health literacy, education, religion) are collected at admission. After discharge, a two-stage medical record review study, based on a Dutch record review study, is conducted by experienced reviewers to determine the incidence of adverse events. Determinants will be analysed by multilevel multivariable stepwise logistic regression. Mechanisms in the care process will be explored in qualitative interviews with patients and providers.

Results Recruitment has started in December, 2010 and will continue for 24 months. Presently, 50 patients were recruited. By August, preliminary data and practical lessons from data collection will be available. 
Conclusion The study will quantify the risk of patient safety incidents among ethnic minority patients in hospital care, unravel the causes, and identify policy opportunities to minimise inequities in care.

\section{P1-367 ADOLESCENT'S ORAL HEALTH: ODONTOLOGICAL NEEDS RAISED BY COMMUNITY HEALTH AGENTS}

doi:10.1136/jech.2011.142976f.59

${ }^{1} \mathrm{M}$ V de Lima Saintrain, * ${ }^{1} \mathrm{C}$ R S Correa, ${ }^{1}$ A P G F Vieira. ${ }^{1}$ Universidade de Fortaleza, Fortaleza, Ceará, Brazil; ${ }^{2}$ Universidade de Campinas, Campinas, São paulo, Brazil

Introduction Epidemiological data are essential for the development of public policies aiming at health promotion and prevention of risk situations, including those that compromise oral health.

Objective To present epidemiological data on adolescents' oral health collected by community health agents (CHA) utilising the Oral Health Community Indicator (ICSB).

Methodology Cross-sectional study conducted in Guaiúba-CE from July 2007 to February 2008. After calibration and training by community health agents, using the ICSB, a total of 743 adolescents were examined. The study was approved by the University of Fortaleza Research Ethics Committee (No. 001/2007).

Results The adolescents had an average of 26.14 \pm 3.61 teeth, 129 $(17.4 \%)$ did not present caries, $301(40.5 \%)$ had one or two, 223 (30.0\%), three or more, 110 (14.8\%) presented residual root, 121 $(25.7 \%)$ tatar, $74(10.0 \%)$ sore gingiva, $15(2.0 \%)$ oral tissues injuries, $49(6.6 \%)$ required prosthesis, 694 (93.4\%) used toothbrush, and 281 $(51.3 \%)$ visited the dentist last year. It was detected an association between the variables: visit to the dentist in the past year $(p=0.0001)$ and gender and between age below 12 and having three or more caries $(\mathrm{p}=0.023)$.

Conclusion The CHA was able to collect relevant data to the elaboration of public policies aiming equity. The assessed adolescents did not reach the goal set by the International Dental Federation, which is, on average, one decayed, missing or filled tooth.

\section{P1-368 PHYSICAL ACTIVITY AND ENVIRONMENT PERCEPTION IN ELDERLY: POPULATION STUDY IN SOUTHERN BRASIL}

doi:10.1136/jech.2011.142976f.60

M W Corseuil, ${ }^{*}$ I J C Schneider, E d'Orsi. Federal University of Santa Catarina, Florianópolis, Santa Catarina, Brazil

Introduction The aim of this study was to evaluate the association between perceived environment and leisure-time physical activity level in a population sample of elderly in a southern capital of Brazil. Methods Cross-sectional study included 1656 elderly (60 years and older) living in the urban area of Florianopolis, Santa Catarina, Brazil in 2009-2010. The leisure-time physical activity was measured using the long International Physical Activity Questionnaire. The 150 min time limit of physical activity per week was used to classify individuals who were active during leisure time. Environmental variables were assessed using a modified version of the Neighbourhood Environment Walkability Scale. Poisson' regression analysis was used to examine the association of environment variables with leisure-time physical activity with calculated prevalence ratios and CIs of $95 \%$ and $p$ values (Wald test) (STATA 9). The analysis took into consideration the clustering of each sample.

Results The prevalence of leisure-time physical activity was $29.7 \%$ (95\% CI 26.0 to 33.3), 35.6\% (95\% CI 29.7 to 41.6) among men and $26.3 \%$ (95\% CI 23.1 to 29.4 ) in women. The existence of sidewalks, bikeways, pedestrian paths, social support from friends/neighbours to motivate physical activities and climate were parameters independently associated with leisure time physical activity.

Conclusion The results suggest that the construction of a safe and enjoyable public environment inside populated urban areas stimulates physical activity practices and thus should be considered Social network can develop and encourage leisure-time physical activities in the society nearby which is especially important for elderly population.

\section{P1-369 THE ASSOCIATION BETWEEN ORAL CONTRACEPTIVE USE AND BONE MASS IN BOTH YOUNG AND OLDER WOMEN}

doi:10.1136/jech.2011.142976f.61

S Wei, ${ }^{*}$ A venn, G Jones. Menzies Research Institute, University of Tasmania, Hobart, Tasmania, Australia

Introduction The association between oral contraceptive use and bone mass remains inconclusive.

Aim To describe the associations between current and ever use of $\mathrm{OC}$, bone mass and vertebral deformity in population-based samples of women.

Methods Data were collected from women aged 26-36 years ( $n=687$ ) in the Childhood Determinants of Adult Health study and aged $50-80$ years $(n=491)$ in the Tasmanian Older Adult Cohort study. OC use was assessed by questionnaire. Bone mass and vertebral deformity were measured by either quantitative ultrasound or dual-energy x-ray absorptiometry.

Analysis Multivariable linear regression analysis was employed to examine the associations between $\mathrm{OC}$ use and bone mass with adjustment for confounders. Logistic regression analysis was used to investigate the associations between $\mathrm{OC}$ use and the presence of vertebral deformity.

Results In young women, current OC use was associated with higher broadband ultrasound attenuation (BUA, $\beta=0.21$ ), speed of sound (SOS, $\beta=0.23$ ) and quantitative ultrasound index (QUI, $\beta=0.23$ ) (all, $p<0.05$ ). The associations remained significant after adjustment except for SOS. In older women, ever use of OC was associated with a higher $\mathrm{BMD}$ at the total body $(6 \%, \mathrm{p}<0.001)$ and spine $(4 \%, p=0.05)$ after adjustment. Longer duration of use was also associated with total body and spine BMD. Consistent reductions in vertebral deformity were observed but these only reached significance for 5 to 10 years use (adjusted OR 0.46, 95\% CI 0.22 to 0.94).

Conclusion OC use is consistently associated with higher bone mass in both young and older women in these population-based samples.

\section{P1-370 LESSONS FOR ZOONOTIC DISEASE AND VECTOR ERADICATION IN NEW ZEALAND FROM PAST SUCCESSES}

doi:10.1136/jech.2011.142976f.62

${ }^{1} \mathrm{~N}$ Wilson, ${ }^{*} \mathrm{D}$ Lush, ${ }^{3} \mathrm{P}$ Bell. ${ }^{1}$ University of Otago, Wellington, New Zealand; ${ }^{2} \mathrm{MAF}$ Biosecurity New Zealand, Wellington, New Zealand; ${ }^{3}$ Department of Conservation, Wellington, New Zealand

Introduction Regional and global disease eradication remains attractive for epidemiologists and policy makers (Science 2010;330:1736-9). New Zealand has successfully eradicated endemic brucellosis and hydatids. It recently eradicated the southern saltmarsh mosquito (SSM), Aedes camptorhynchus, a vector for Ross River virus. In some areas of the country bovine tuberculosis appears to have been eliminated via various control measures relating to the brushtail possum (Trichosurus vulpecula). 